\title{
Un outil de reconnaissance géophysique en milieu urbain : la prospection électrostatique
}

\section{A. TABBAGH}

UMR 7619 Sisyphe département de Géophysique appliquée Université Pierre-et-MarieCurie (Paris 6) case 105

4. place Jussieu 75252 Paris Cedex 05 Institut de sciences et technologie géophysique-géotechnique case 95

4. place Jussieu 75252 Paris Cedex 05 alat@ccr.jussieu.fr

C. PANISSOD C. BENECH M. DABAS A. JOLIVET R. GUÉRIN

UMR 7619 Sisyphe département de Géophysique appliquée Université Pierre-et-Marie-

Curie (Paris 6) case 105

4, place Jussieu 75252 Paris Cedex 05
La prospection géophysique en milieu urbain souffre de différentes difficultés : vibrations, bruits électrique et électromagnétiques, structure superficielle complexe et présence d'ouvrages. Pour explorer les dix premiers mètres, la méthode électrostatique peut remplacer la méthode électrique à courant continu pour fournir une image des structures du sous-sol en utilisant les mêmes outils d'interprétation.

Trois exemples d'emploi de cette méthode sont présentés. Le premier correspond à la caractérisation du milieu dans lequel le microtunnel doit être foré. Le deuxième étudie le pavement de la cathédrale de Bastia en vue de connaître la structure de l'église médiévale. Le troisième permet de positionner l'emplacement de la muraille antique d'Alexandrie dans le sous-sol des rues de la ville actuelle.

Mots-clés : quadripôle électrostatique, résistivité électrique, reconnaissance géophysique, milieu urbain.

\section{Electrostatic prospecting: a tool for geophysical survey in urban context}

Geophysical survey in urban context suffers from a series of external factors: vibrations, electrical and electromagnetic nolses, complex subsurface structure and presence of buildings. To explore the first ten metres, the electrostatic method can replace the DC electrical method to image the structure of the ground with the same interpretation methods. Three examples are presented hereafter. In the first, one attempted to characterise the medium in which a micro-tunnel had to be drilled. In the second, one looks under the Bastia cathedral pavement, for the preceding medieval church remains, In the third, the path of the antique wall, a major feature of the global organisation of the city of Alexandria, was located under the streets:

Key words : electrostatic four poles array, electrical resistivity, geophysical survey, urban medium. 


\section{Introduction}

La reconnaissance géophysique a pour caractéristiques d'être non destructrice, de pouvoir être réalisée selon un échantillonnage spatial suffisamment fìn et d'être rapide. En revanche, elle a pour inconvénient le fait de ne pas porter directement sur le (ou les) paramètre mécanique qui permet une prise de décision par le géotechnicien. Elle s'applique a priori aussi bien en milieu urbain qu'en milieu rural, mais le premier présente une série de difficultés particulières que l'on peut résumer ainsi

- il y a en ville des sources de bruits : vibrations, courants électriques vagabonds, mouvements de véhicules...

- le sous-sol peut y ètre plus complexe, y présenter. en particulier, des structures très contrastées toutes proches de la surface

- l'accès à la surface à explorer y est plus limité, par la présence de bâtiments notamment.

Les méthodes géophysiques et leurs procédures de mise en cuvre doivent donc être adaptées aux contraintes spécifiques du milieu urbain.

La résistivité électrique, propriété qui présente la plus large gamme de variations et qui est particulièrement sensible aux variations de teneur en argile et d'humidité, est difficile à mesurer en ville. Les méthodes électromagnétiques basse fréquence, dont la mise en œuvre est la plus rapide, sont en effet très sensibles aux masses métalliques (voitures, armatures du béton, câbles...) et la méthode électrique, très peu sensible à ce type de perturbations, requiert par contre la mise en place d'électrodes ce qui n'est pas réalisable sur les sols construits (bitume, pavage, dalle de ciment...). La méthode électrostatique, introduite à l'origine pour l'exploration peu profonde de milieux très secs, évite l'utilisation d'électrodes tout en gardant la faible sensibilité aux bruits de la méthode électrique, elle est donc potentiellement très intéressante en milieu urbain. Après avoir présenté son principe et les appareils utilisés, trois exemples de prospection en milieu urbain seront développés.

\section{Principe et limites de la méthode}

La méthode électrostatique peut être présentée comme une généralisation de la méthode électrique (Grard et Tabbagh, 1991) où quatre pôles électrostatiques, placés dans l'air au-dessus du sol, sont utilisés pour injecter le courant et pour mesurer la différence de potentiel résultante. Mais on ne peut pas travailler à la fréquence zéro où le maintien de charges électrostatiques sur les pôles serait impossible ; on utilise donc une injection en courant alternatif. Quand un tel quadripôle est posé sur le sol et que la fréquence utilisée est suffisamment basse, les résultats et les méthodes d'interprétation sont les mêmes que pour la méthode électrique.

La limite en fréquence peut être fixée (Benderitter et al. 1994; Tabbagh et Panissod, 2000) à partir du nombre d'induction qui doit rester inférieur à 0,1 environ. Ce nombre d'induction compare la taille du qua- dripôle à la profondeur de pénétration caractérisant l'effet de peau (c'est-à-dire l'atténuation du signal avec la profondeur, et la décroissance de la profondeur d'investigation avec la fréquence du signal). Si, par exemple, la distance entre un pôle d'injection et le plus proche pôle de mesure est L, la fréquence f, et la conductivité électrique moyenne du terrain $\sigma$, on aura une valeur du nombre d'induction : $\sigma \mu 2 \pi \mathrm{fL}^{2}$ inférieure à $0,1 \mathrm{si} \mathrm{f}$ reste inférieure à $12 \mathrm{kHz}$ pour $\mathrm{L}=10 \mathrm{~m}$ et $\sigma=$ $0,01 \mathrm{~S} / \mathrm{m}$ ( $\mu$ est la perméabilité magnétique du milieu égale à celle du vide, $4 \pi 10^{-7} \mathrm{H} / \mathrm{m}$, dans la plupart des sols).

En pratique, les pôles sont constitués par des pièces métalliques de formes quelconques; on peut par exemple utiliser des plaques métalliques de dimension linéaire petite devant l'écartement entre les pôles. L'impédance des pôles d'injection est inversement proportionnelle à leur surface et à la fréquence. En général on reste au-dessus de $10 \mathrm{kHz}$, ce qui conduit à une profondeur d'investigation de la dizaine de mètres pour les résistivités les plus courantes.

Plusieurs appareillages ont été réalisés au cours des dix dernières années. La figure 1 montre le multipôle ( routier »), MPR, qui comprend un dipôle d'injection et deux dipôles de mesure qui permettent deux profondeurs d'investigation différentes. Dans ce dispositif, les pôles sont placés à l'intérieur des roues et le multipôle, tiré par un véhicule léger, peut ètre utilisé dans les rues et sur routes. Les mesures sont enregistrées à un pas de 10 à $20 \mathrm{~cm}$ et la vitesse de progression est celle du véhicule. Ce système (Panissod et Tabbagh, 1996 ; Panissod et al., 1998) a été développé avec le soutien de la FSTT (Comité Français des Travaux sans Tranchées). Un deuxième dispositif composé de pôles indépendants (Fig, 2) de surface 0,5 ou $1 \mathrm{~m}^{2}$ permet de réaliser des sondages jusqu'à des distances entre pôles d'une trentaine de mètres.

\section{3}

\section{Reconnaissance de la rue de Mulhouse à Champigny-sur-Marne (Val-de-Marne)}

Cette reconnaissance a été réalisée à la demande de la Direction des services de l'eau et de l'assainissement (DSEA) du Val-de-Marne, en préalable à un chantier de microtunnelier pour la mise en place d'un collecteur Les mesures réalisées le long de la rue de Mulhouse à Champigny-sur-Marne, sur deux profils parallèles ont montré un milieu latéralement homogène (Fig. 3) où les variations latérales sont de faible amplitude et ont une origine superficielle puisqu'elles apparaissent sur le plus petit quadripôle et sont de courtes longueurs d'onde spatiales. Ces profils montrent que la résistivité varie de façon importante avec la profondeur : on a une résistivité apparente proche de $150 \Omega$.m pour le quadripôle métrique, $70 \Omega$ m pour le quadripôle de $2 \mathrm{~m}$ et $20 \Omega \mathrm{m}$ pour le quadripôle à pôles indépendants écartés de $4 \mathrm{~m}$. Cette variation est confirmée par le sondage électrostatique (Fig. 4) qui montre l'existence d'une épaisse couche d'argile, entre 1,1 et $5,5 \mathrm{~m}$ si on lui donne une résistivité de $8 \Omega . \mathrm{m}$, et qui serait plus épaisse si on augmente cette résistivité (loi d'équivalence clas- 

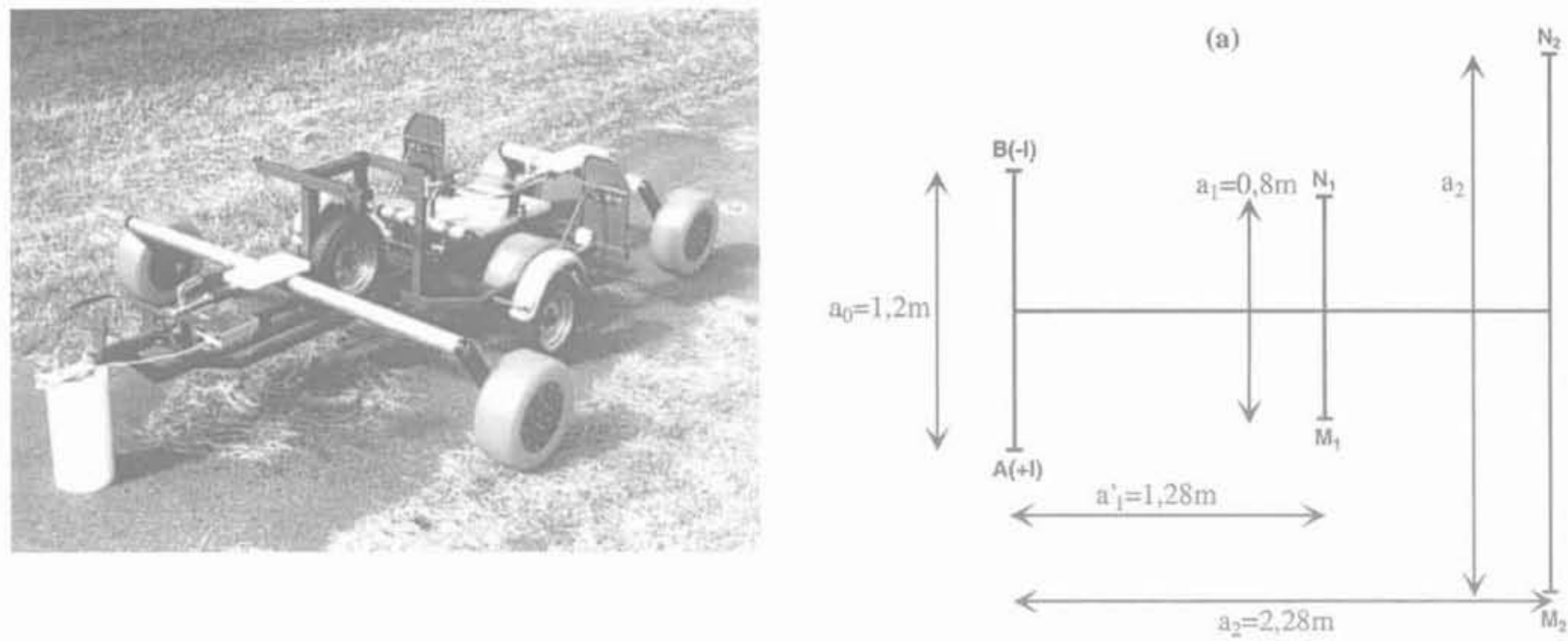

FG.1. Multipôle électrostatique « routier » MPR (a : photo ; b : schéma).

Electrostatic multipole MPR is for road studies in (a : photo; b: schema).
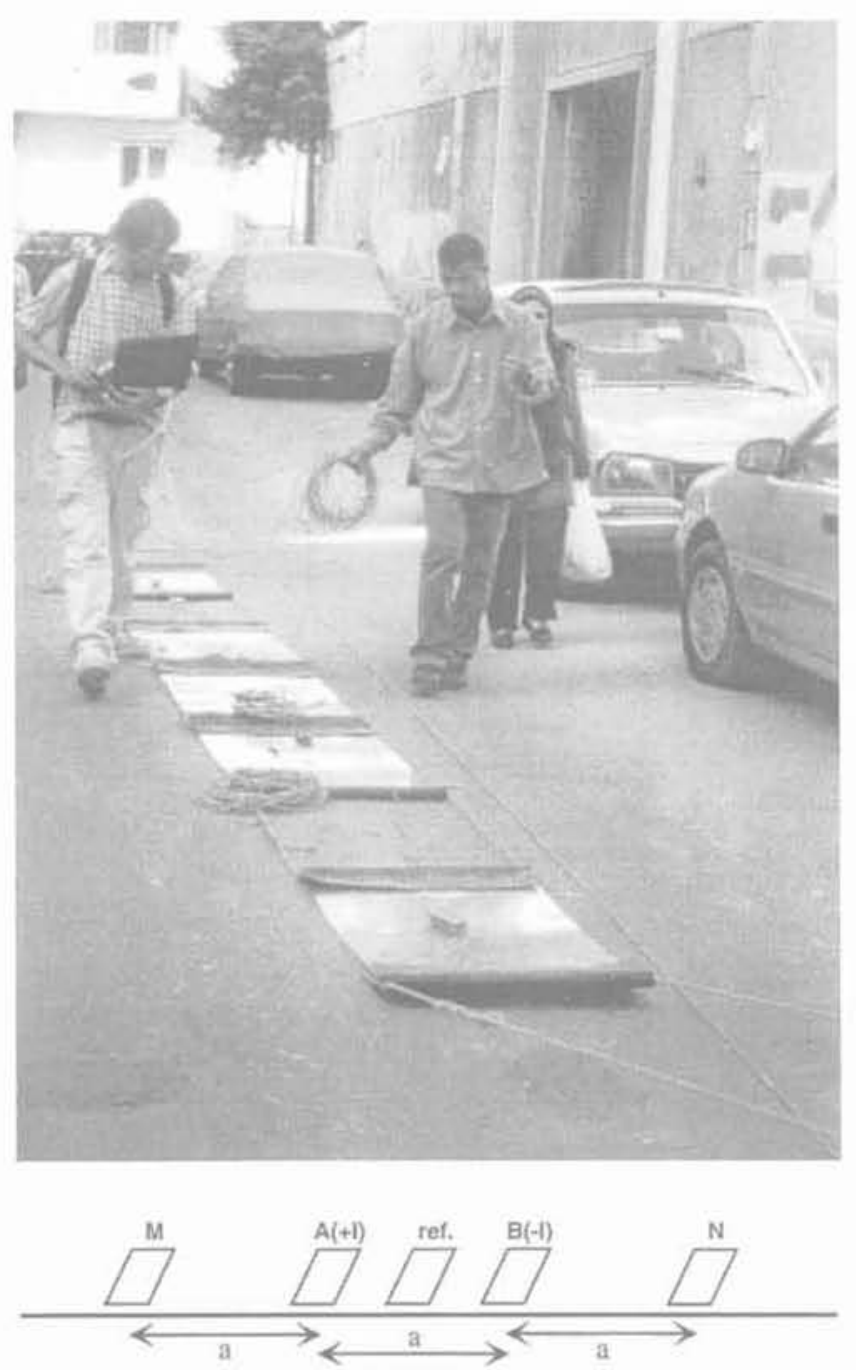

FIG. Q Quadripôle électrostatique avec pôles indépendants ( $\mathrm{a}$; photo ; $\mathrm{b}$; schéma). Electrostatic four poles array with independent poles (a: photo; $b$ : schema). sique en méthode électrique spécifiant que les propriétés d'un terrain conducteur encadré au-dessus et en dessous par des terrains résistants ne sont pas déterminées de façon unique, mais au contraire que le rapport épaisseur sur résistivité est constant, tant que l'épaisseur n'est pas trop importante). Avec cette expérimentation, le milieu dans lequel devait être implanté l'ouvrage a été bien caractérisé.

\section{Étude du sous-sol de la cathédrale Sainte-Marie de Bastia}

L'étude, demandée par la Direction du patrimoine de la ville de Bastia (Corse) dans le cadre d'un programme européen PROGRESS, visait à reconnaitre le sous-sol de la cathédrale afin de déterminer l'existence d'édifices antérieurs. Pendant la première visite sur le terrain, on nous avait signalé l'existence d'affaissements dans le dallage pour une cause alors inconnue. L'objectif secondaire était donc de comprendre l'origine de ces effondrements qui avaient nécessité une réfection des scellements du dallage à période régulière.

La cathédrale actuelle a été bâtie entre 1604 et 1670 sur les vestiges de l'ancienne église Santa Maria della Consolazione datant de la fin du XVe siècle. Cette église fut en effet jugée trop petite à partir du moment oú Terra nova, la cité génoise enfermée dans les remparts de Bastia, fut construite. Elle reprend le plan d'une nef à trois vaisseaux ( $45 \mathrm{~m} \times 25 \mathrm{~m} \times 17 \mathrm{~m}$ ).

L'église primitive est connue grâce aux sources écrites : la construction de cette église se situe autour de 1490 (une indulgence est accordée en 1488 pour la construction de l'église, un curé est nommé en 1489 et la cloche arrive d'Italie en 1491). Cette église est petite, à nef unique et est orientée nord-sud donc perpendiculairement à la cathédrale actuelle. Sa longueur est de 


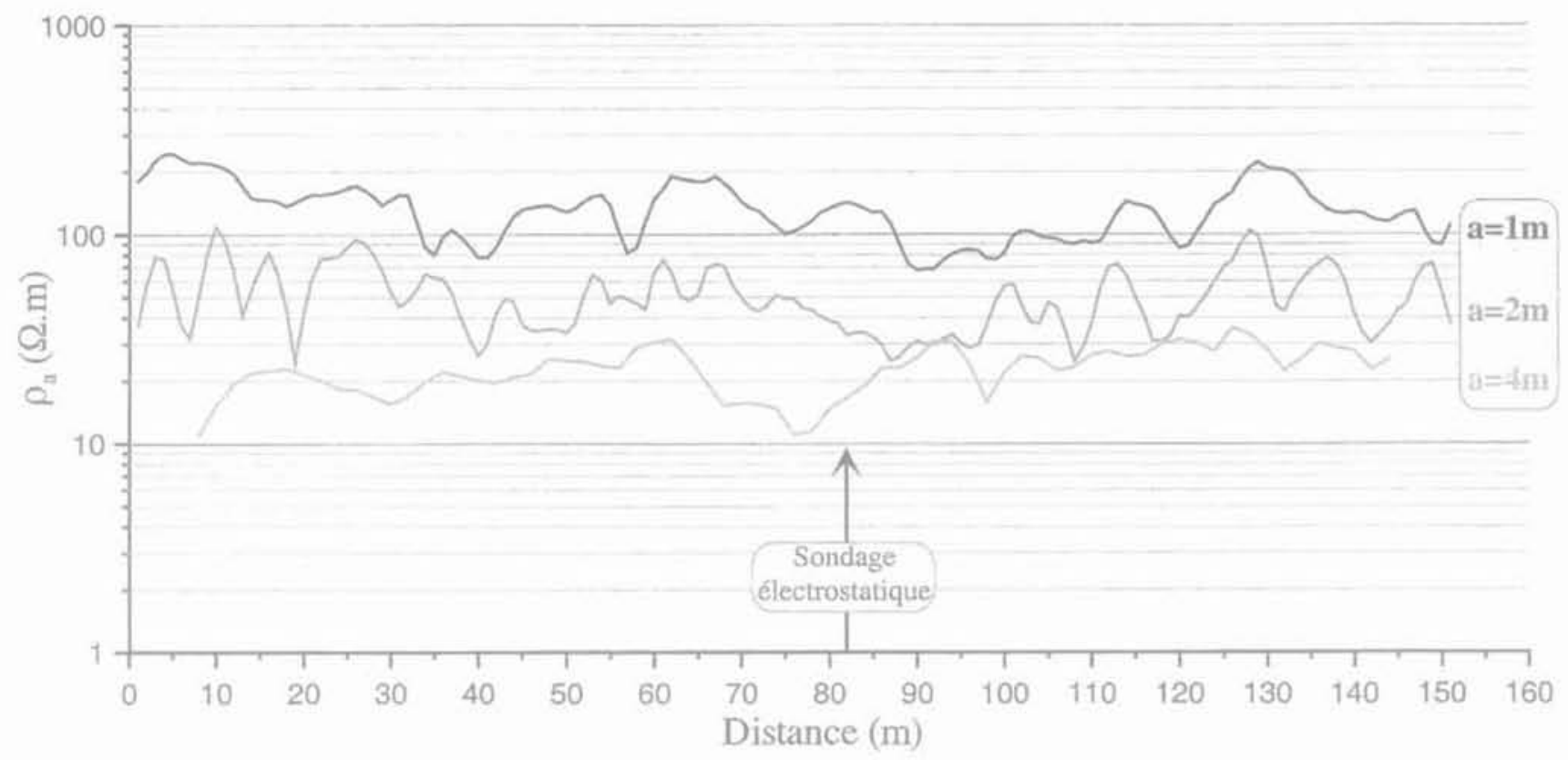

Fig. 3 Profils électrostatiques Wenner dans la rue de Mulhouse (du $n^{\circ} 140$ au $n^{\circ} 230$ ) à Champigny-sur-Marne. Wenner electrostatic profiles in Mulhouse street (between $n^{\circ} 140$ and $n^{\circ} 230$ ) at Champigny-sur-Marne.

l'ordre de $13 \mathrm{~m}$ (une poutre de faîte de cette longueur est commandée en 1491). Cette église fut démolie au début du XVI" siècle, et le rocher sur lequel elle s'appuyait devint une carrière pour les bâtisseurs.

Une prospection électrostatique visant à retrouver des éléments de l'église du XVe siècle a été effectuée dans la nef et les deux collatéraux sur toute la surface disponible. Nous avons procédé avec un appareil ayant des pôles réglables et donc un encombrement restreint, à des mesures suivant deux directions : vingt-huit profils dans la direction est-ouest (longueur maximale $24,2 \mathrm{~m}$ ) et vingt-sept profils dans la direction nord-sud

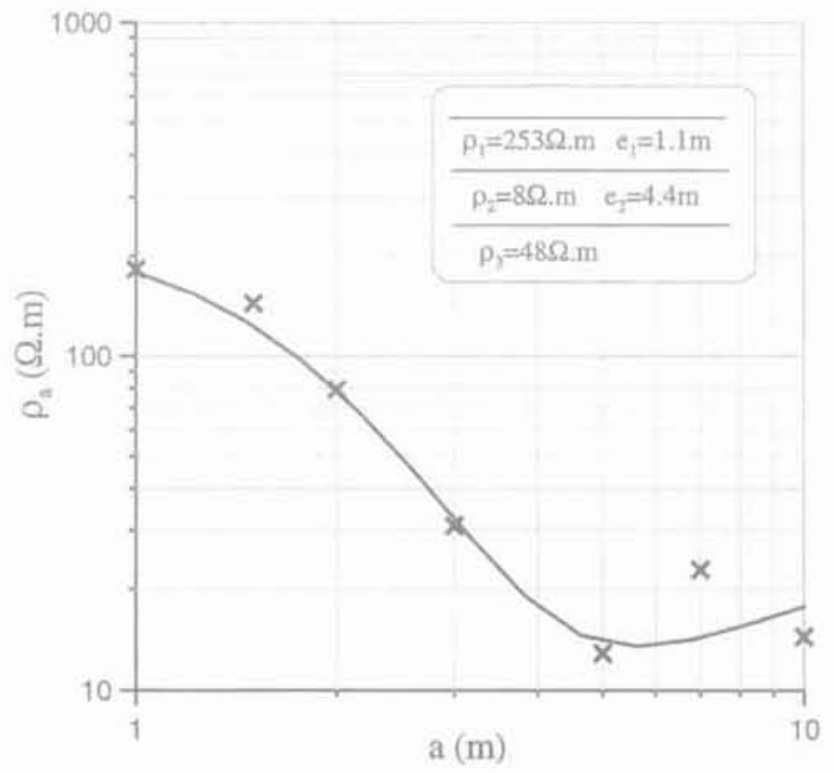

FG.4 Sondage électrostatique Wenner positionné sur la figure 3 .

Wenner electrostatic sounding located in figure 3 . (longueur maximale 16,6 m). Les profils sont espacés de $0,5 \mathrm{~m}$ et la distance entre deux mesures est de $0,1 \mathrm{~m}$.

Une prospection radar à la fréquence $450 \mathrm{MHz}$, a été réalisée avec le même maillage mais a permis de s'approcher plus près des piliers et contreforts (faible encombrement de l'antenne). Les profils ont été assemblés entre eux. La méthode radar permet d'extraire une information en fonction de la profondeur et donc de «couper » le terrain en tranches (à l'inverse de l'électrostatique qui intègre le signal entre la surface du sol et la profondeur d'investigation du dispositif choisi). Nous avons effectué ce traitement par tranches d'épaisseur de $20 \mathrm{~cm}$ en partant de la profondeur de $30 \mathrm{~cm}$ jusqu'à $1,7 \mathrm{~m}$. Au-delà, l'onde radar n'est plus assez énergétique pour pénétrer.

Les cartes électrostatiques obtenues avec le petit et le grand quadripôle électrostatique (Figs. 5 et 6 ) montrent très nettement l'existence d'une masse résistante au nord de direction est-ouest (numéroté 1 sur les figures). Plusieurs structures moins résistantes apparaissent sur les côtés nord et sud. Tout le centre de la cathédrale se traduit par une masse conductrice assez. homogène. Une anomalie résistante (numérotée 2 sur les figures) se voit en revanche très nettement entre le premier et le second pilier au nord du collatéral sud. Cette anomalie est encore plus visible sur la carte correspondant à la plus grande profondeur d'investigation, elle s'étend donc en profondeur (>1 m).

Sur la carte radar (Fig. 7) correspondant au terrain superficiel (tranche de 4 à $8 \mathrm{~ns}$, soit à environ $0,3 \mathrm{~m}$ ) présentée suivant une échelle normalisée, les zones en noir/gris foncé correspondent aux zones réfléchissantes (présence de réflecteurs comme un ancien sol, une interface roche-mère/remplissage...) et les zones en blanc/gris clair à des zones homogènes qui laissent passer l'onde radar. On remarque la présence systématique de deux bandes nord-sud réfléchissantes associées à chacun des piliers du collatéral sud. Une autre bande est-ouest, moins visible, semble aussi relier tous 


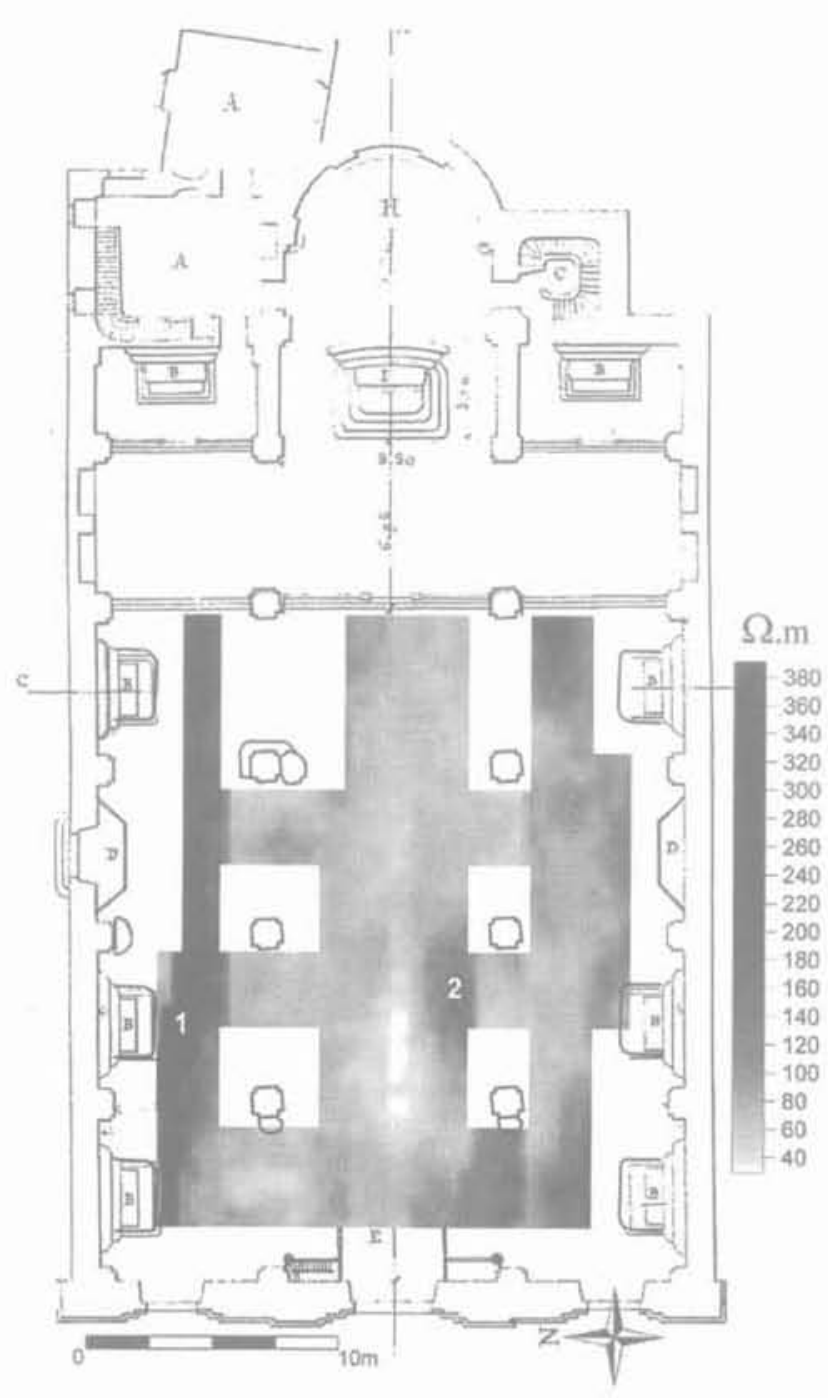

AG.5 Prospection électrostatique avec le petit quadripôle $(\mathrm{a}=0,60 \mathrm{~m})$ dans la cathédrale Sainte-Marie de Bastia.

Electrostatic survey with small four poles array $(\mathrm{a}=0.60 \mathrm{~m})$ in Sainte-Marie cathedral at Bastla.

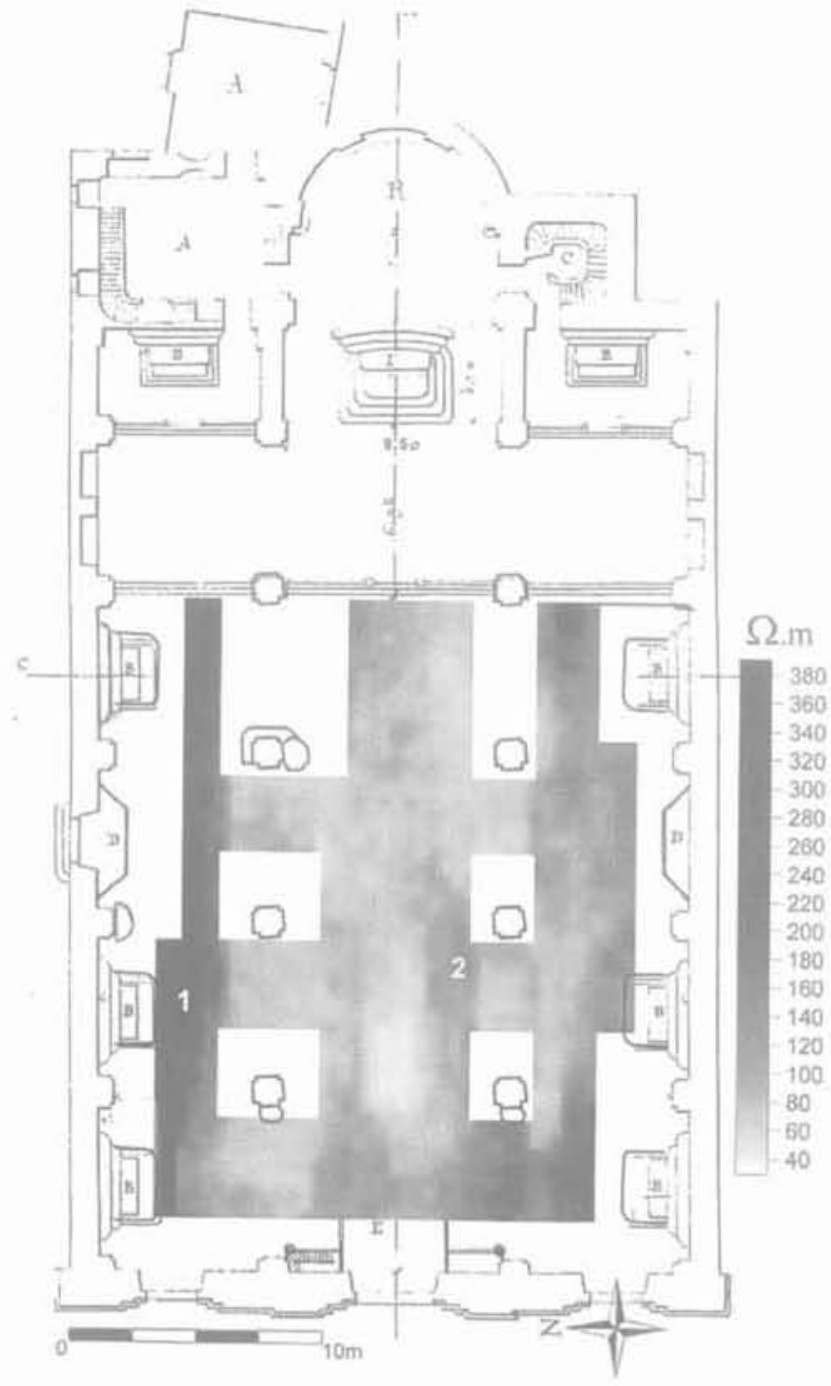

FG.6 Prospection électrostatique avec le grand quadripôle $(\mathrm{a}=1,50 \mathrm{~m})$ dans la cathédrale Sainte-Marie de Bastia.

Electrostatic survey with main four poles array $(\mathrm{a}=1.50 \mathrm{~m})$ in Sainte Marie cathedral at Bastia. les piliers de ce collatéral. Pour le collatéral nord, deux bandes associées au pilier le plus à l'est (le plus proche du chceur) sont visibles. Ceci est moins évident à proximité des deux autres piliers. Les autres cartes radar plus profondes $(0,5,0,7$ et $0,9 \mathrm{~m})$, non présentées ici, sont beaucoup plus homogènes traduisant l'homogénéité du remplissage, mis à part une anomalie résistante au nord. A partir de $1,1 \mathrm{~m}$, de nombreux réflecteurs apparaissent sans organisation spatiale apparente. Cette profondeur correspond à un changement important des propriétés physiques du sol.

L'interprétation des données radar vient confirmer les données électrostatiques et les compléter. Sur la carte radar, nous pensons que les bandes réfléchissantes correspondent à un chainage entre les différents piliers. Le fait que nous ne les voyons clairement que dans la partie sud a probablement une signification que nous ne connaissons pas (mais nous ne pouvons pas dire s'il en existe ou non sous le collatéral nord).
Il est probable que les zones résistantes (en noir/gris foncé sur la carte électrostatique) correspondent à la roche-mère quasi affleurante. Ces zones refléteraient donc l'emplacement du rocher sur lequel est construite la cathédrale, ce qui est corroboré par l'ancien nom de I'église (Santa Maria l'arrembata) et une chronique. Il y a une différence importante entre le côté nord très abrupt et le côté sud où la variation est beaucoup plus progressive. Peut-être peut-on y voir la différence entre une roche proche de la surface en amont et un remblai plus important en aval, au sud, vers la mer. De ce changement très rapide du matériau constituant le substrat dans le collatéral nord proviennent certainement les problèmes de stabilité signalés à plusieurs reprises. De plus, il semble logique que le chaînage présent dans la partie sud soit moins utile sous le collatêral nord du fait de la présence d'une assise solide sur le rocher.

Il n'est pas possible au vu des données géophysiques de préciser l'emplacement de l'église de direc- 


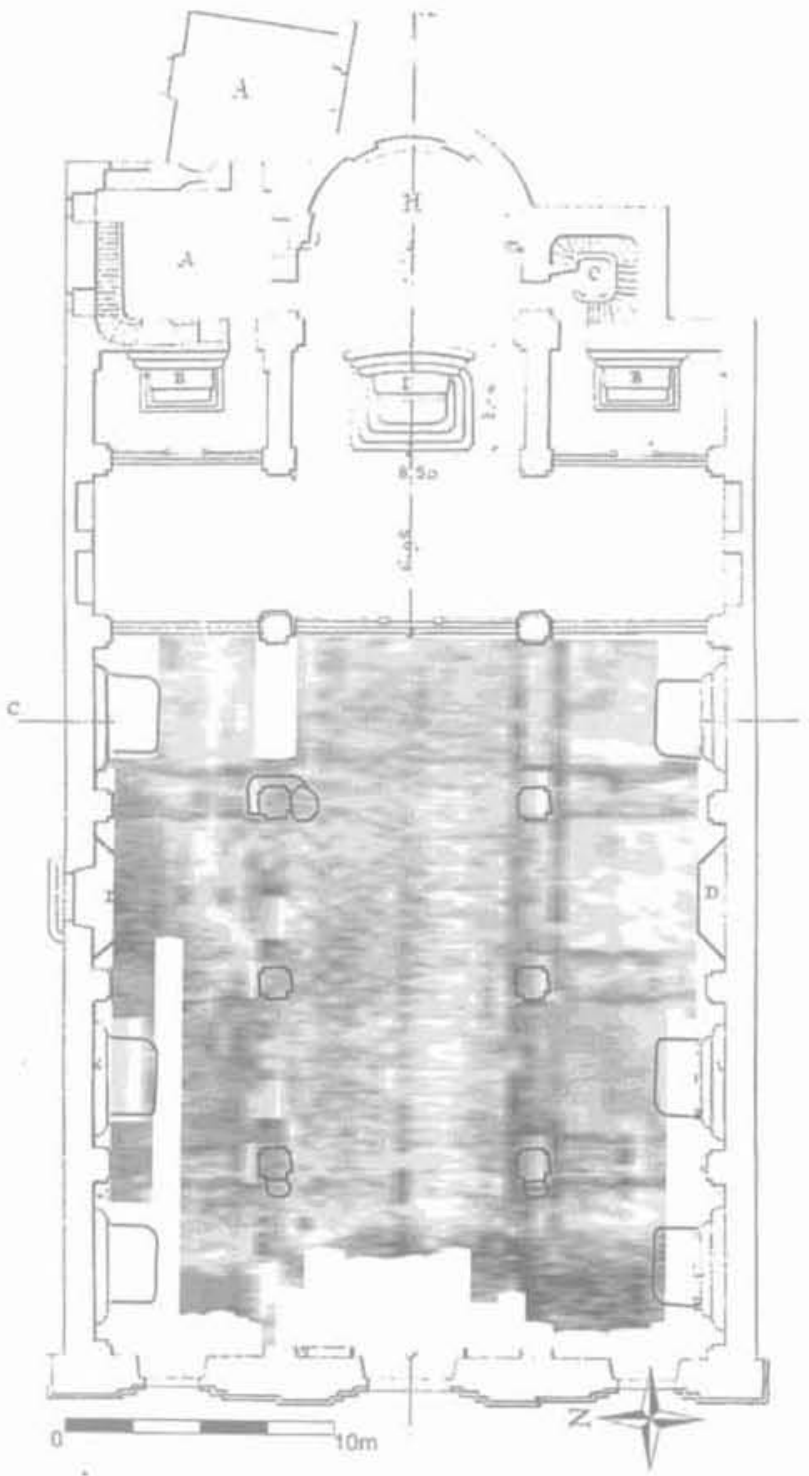

FIG. 7 Coupe entre 4 et 8 ns issue de la prospection radar $(450 \mathrm{MHz})$ dans la cathédrale Sainte-Marie de Bastia. Time slice between 4 ns and 8 ns from radar survey $(450 \mathrm{MHz})$ in Sainte Marie cathedral at Bastia.

tion nord-sud. La masse rocheuse au nord peut constituer une limite à son extension mais nous ne pouvons définir sa limite au sud. Comme la roche remonte à l'ouest du premier pilier ainsi qu'à l'est du troisième pilier, on pourrait placer l'église entre le premier et le troisième pilier. Il ne faut pas oublier que si l'emplacement a servi de carrière, il n'est pas étonnant non plus de ne pas voir de trace claire de l'église du XVe siècle.

Nous avions recommandé dans le rapport (rédigé en novembre 1997) de réaliser un sondage archéoloqique à l'aplomb de l'anomalie résistante (numérotée 2 sur les figures 5 et 6 ) entre le premier et le second pilier sud de la cathédrale qui correspondrait à un vide de grande dimension (minimum $1 \mathrm{~m} \times 3 \mathrm{~m}$ ) et proche de la surface. Avant ce contrôle, un effondrement a eu lieu en 1998. On y a trouvé un ossuaire dont le tassement a créé le vide détecté par les méthodes géophysiques. Ces ossuaires sont souvent " clandestins » : les inhumations ayant été interdites dans les édifices religieux, les
Bastiais (comme d'autres Européens de l'époque) procédaient à une inhumation secondaire de leurs morts clandestinement la nuit dans la cathédrale.

En conclusion, la localisation des affleurements rocheux sous la cathédrale Sainte-Marie, donc la forme du remblaiement réalisé pour les fondations de la cathédrale, a été cartographiée. La découverte d'anomalies radar liées à un chaînage des piliers est un résultat important. La dissymétrie entre la profondeur de la roche entre les deux collatéraux explique les problèmes de stabilité de l'édifice. De l'église antérieure, rien n'est visible, conséquence probable de l'exploitation en carrière après sa destruction. Enfin, une anomalie correspondant à un vide important a été vérifiée par sondage à l'aplomb des piliers du collatéral sud.

\section{5}

\section{Recherche du tracé de la muraille antique d'Alexandrie (Égypte)}

Le projet concernant la recherche des murailles antiques d'Alexandrie a débuté en octobre 2000 par l'investigation de plusieurs rues avec un système électrostatique à pôles indépendants. Le choix de cette méthode a été motivé par les résultats obtenus lors d'une précédente mission à Alexandrie en 1997, dirigée par Albert Hesse, et qui concernait la recherche du tracé de l'Heptastade, i.e. la digue d'environ $1 \mathrm{~km}$ de long (7 stades grecs) qui reliait la ville à l'île de Pharos sur laquelle se trouvait le phare d'Alexandrie (Hesse et al., 1998). Parmi les différentes méthodes géophysiques utilisées (radar, sismique, électromagnétisme), l'électrostatique s'est révélée être la plus appropriée pour l'exploration du sous-sol alexandrin où les niveaux antiques se situent dans les premiers mètres de profondeur. Les résultats de cette prospection avaient permis de reconstituer le scénario de formation de l'isthme d'Alexandrie, reposant sur l'ancienne digue que constituait l'Heptastade et qui séparait les deux ports de la ville.

Pour l'Heptastade, la méthode électrostatique avait permis de mettre en évidence un phénomène de grande échelle, à savoir l'élargissement de l'isthme par apports successifs de sédiments par la mer. Le projet sur les murailles impliquait une recherche plus fine, plus exigeante, puisqu'elle concernait l'identification d'une structure archéologique bien définie. Du point de vue archéologique, les connaissances concernant l'identification du tracé de la muraille antique sont maigres : Mahmoud Bey, un chercheur égyptien, a réalisé dans la seconde moitié du XIX siècle une série de sondages qui a permis de reconnaitre les fondations de l'enceinte en cinq endroits différents dans sa partie méridionale. Le reste du tracé est encore aujourd'hui sujet à controverses, particulièrement à l'est oủ l'on ne sait pas exactement jusqu'où s'étendait la ville dans cette direction. Les fondations dégagées se trouvaient à environ 3-4 m de profondeur : larges de $5 \mathrm{~m}$, elles étaient constituées de deux parements en gros blocs probablement calcaires, et d'un remplissage de cailloux liés au mortier. L'aspect massif de ces structures pouvait laisser espérer que malgré les perturbations dues aux occupations modernes, il était possible de détecter leur présence par la réalisation de panneaux électriques 


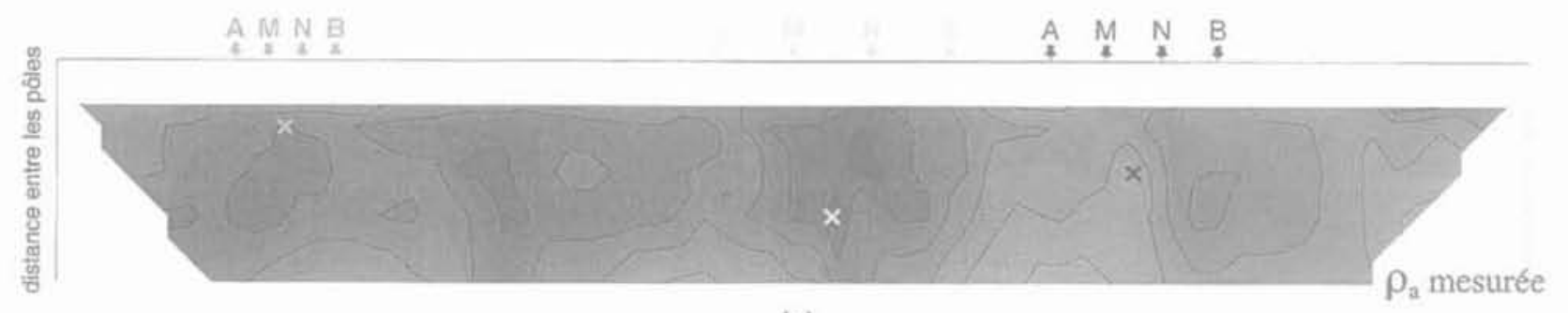

erreur $\mathrm{RMS}=13 \%$

(a)
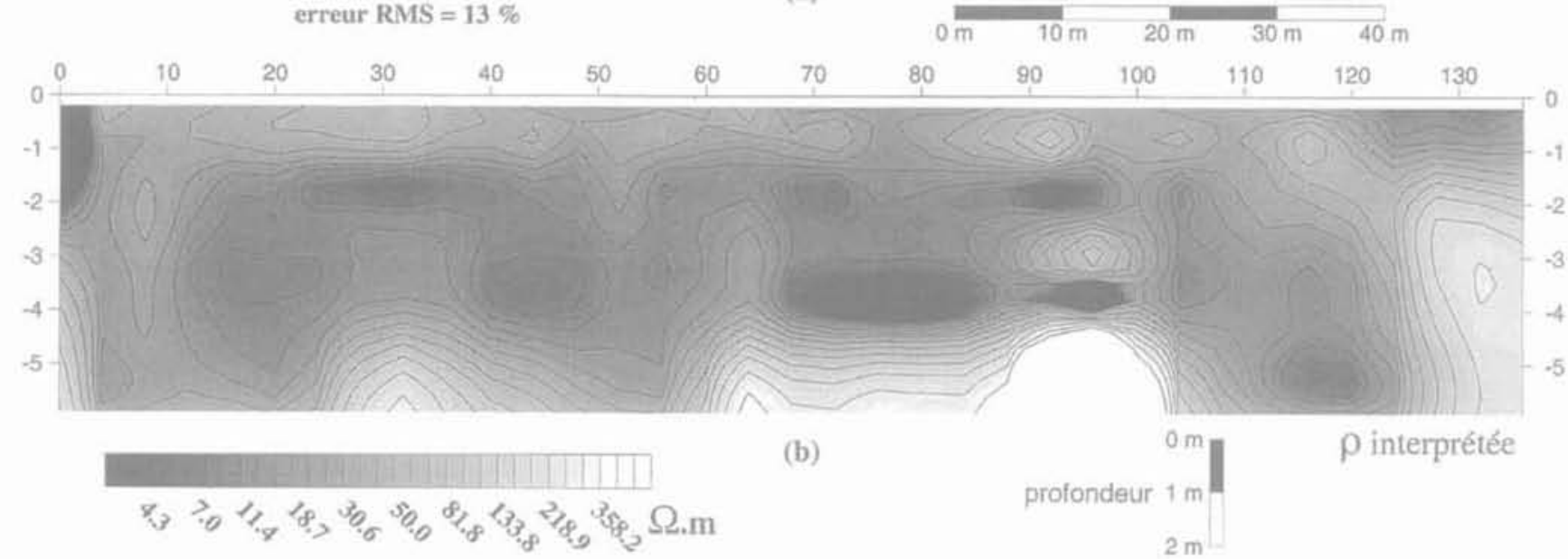

FiG. 8 Panneau électrique (a : convention de représentation de la résistivité électrique apparente mesurée ; b : modèle de résistivité électrique interprétée) obtenue pour la recherche du tracé de la muraille antique d'Alexandrie (Égypte).

Electrical 2D-imaging (a : visualisation mode of measured apparent electrical resistivity : $b$ : interpreted electrical resistivity model) obtained for the research about the location of the antique walls of Alexandria (Egypt).

avec des pôles électrostatiques indépendants. Ces panneaux sont obtenus après plusieurs passages avec des quadripôles à écartement de plus en plus grand (distance entre deux pôles successifs de $2,3,5,7$ et $10 \mathrm{~m}$ ). Ils permettent de mesurer à différentes profondeurs la résistivité électrique apparente le long d'un même profil, et de visualiser les résultats sous la forme conventionnelle d'une pseudo-section (Fig. 8a). La figure 8b présente les variations latérales et verticales de la résistivité électrique interprétée obtenues par inversion avec le logiciel Res2dinv (Loke et Barker, 1996).

La première mission avait pour objectif de « validern l'utilisation de la méthode électrostatique et d'identifier la " signature géophysique » des fondations de la muraille. Les premiers essais ont porté sur la partie méridionale dont le tracé est à peu près localisé grâce aux travaux de Mahmoud Bey. Les panneaux électriques, de longueurs différentes, ont été réalisés dans six rues dont le tracé recoupe perpendiculairement celui supposé de la muraille. Le profil électrostatique présenté ici a permis de localiser une anomalie résistante, située à l'abscisse $95 \mathrm{~m}$, à environ $4 \mathrm{~m}$ de profondeur et large de $5 \mathrm{~m}$ (Fig. 8), à proximité du tracé proposé par Mahmoud Bey. Ces résultats demandent maintenant à être confirmés par d'autres investigations qui montreront que cette anomalie signale effectivement la présence des fondations de la muraille et permettront de reconnaitre la limite orientale de la ville dans l'Antiquité.

\section{6}

\section{Conclusion}

La méthode électrostatique est une alternative efficace à la méthode électrique classique. Elle permet d'acquérir dans les milieux urbains des informations sur les variations latérales et verticales de la résistivité électrique, paramètre adéquat et sensible en géotechnique. Son emploi sur du bitume (prospections pour le positionnement d'un microtunnel à Champigny-sur-Marne, et pour la localisation d'une muraille antique à Alexandrie) ou des dallages (prospection pour la reconnaissance de l'édifice antérieur à la cathédrale Sainte-Marie de Bastia), apporte des informations sur la structure du sous-sol, avec des mesures rapides d'acquisition, peu coûteuses et non destructrices.

L'utilisation de ce type d'appareil ouvre donc de larges perspectives dans l'exploration du sous-sol urbain et peut aider à mieux analyser en amont les problèmes liés à ce milieu extrêmement complexe, que ce soit en génie civil ou en archéologie, et sélectionner de façon plus pertinente les emplacements pour des travaux d'aménagements ou d'étude.

\section{REMERCIEMENTS}

Les auteurs tiennent à remercier la FSST (Comité français des travaux sans tranchées), le programme européen PROGRESS, et le Centre d'études alexandrines. 
Benderitter Y., Jolivet A., Mounir A., Tabbagh A. - "Application of the electrostatic quadripole to sounding in the hectometric depth range 8 . Journal of Applied Geophysics. 31, 1994, p, 1-6.

Grard R. Tabbagh A. - " A mobile four electrode array and its application to the electrical survey of planetary ground at shallow depths x. Journal of Geophysical Research. 96, 1991, p. 4117-4123.

Hesse A., Andrieux P., Atya M. Benech C. Camerlynck C. Dabas M.. Féchant C. Jolivet A., Kuntz C.. Mechler P., Panis- sod C., Pastor L., Tabbagh A., Tabbagh J, - « Geophysical investigations for the location of the Heptastadium in Alexandria (Egypt) 1. Proceedings of the 4th. meeting of the Environmental and Engineering Geophysical Society, Barcelona (Spain), A. Casas ed., 1998, p. 715-718 Loke M.H., Barker R.D. - * Rapid least. square inversion of apparent resistivity pseudosections by a quasi-Newton method n. Geophysical Prospecting, 44 . 1996, p. 131-152.

Panissod C., Dabas M. Hesse A., Jolivet A..
Tabbagh J., Tabbagh A. - "Recent development in shallow depth electrical and electrostatic prospecting using mobile arrays n. Geophysics, 63, 1998, p. $1542-1550$

Panissod C., Tabbagh A. - Etude d'un système multipóle électrostatique. Étude $n^{4} 9$, rapport de thèse $n^{\circ} 7$, Projet national microtunnel, FSTT, 1996.

Tabbagh A. Panissod C. - " 1D complete calculation for electrostatic soundings interpretation 1. Geophysical Prospecting. 48, 2000, p. 511-520. 